\title{
The Biocompatibility Manifesto: Biocompatibility for the Twenty-first Century
}

\author{
Buddy D. Ratner
}

Received: 26 March 2011 / Accepted: 23 May 2011 / Published online: 28 June 2011

(C) Springer Science+Business Media, LLC 2011

\section{Introduction-The Challenge}

The eighteenth and nineteenth centuries were shaped by steam power and industrialization. The twentieth century saw electricity and nuclear energy. Most prognosticators posit that the twenty-first century will be the century of biology. Concepts of biocompatibility were developed at about the same time that cytokines and cell surface receptors were discovered, and these seminal discoveries were never integrated into early biocompatibility definitions. Now is the appropriate time, the century of biology, for modern, quantitative biology to be directed toward improving biocompatibility and toward appropriately defining our improved bioreactions.

With this manifesto, I challenge the definitions for biocompatibility that we use today. I reinforce this challenge by demonstrating why advances in biology and materials have diminished the value of older definitions. Finally, I propose a new definition for biocompatibility that is consistent with new discoveries from biology and recent demonstrations of improved healing of biomaterials implanted in living organisms.

\section{Origins and Definitions}

A number of papers appeared in the 1940s, 1950s, and 1960s examining the tissue reaction to implanted biomaterials

Manifesto: A public declaration of principles and intentions

B. D. Ratner $(\bowtie)$

University of Washington Engineered Biomaterials (UWEB21),

University of Washington,

Seattle, WA 98195, USA

e-mail: ratner@uweb.engr.washington.edu (for example, see [1]). Though many of these early observations were accurate, the word "biocompatible" was never used. By 1970, a few papers appeared that used the word "biocompatible." According to the biocompatibility article in Wikipedia, "The word biocompatibility seems have been mentioned for the first time in peer-review journals and meetings in 1970 by RJ Hegyeli (Amer Chem Soc Annual Meeting abstract) and CA Homsy et al. (J Macromol Sci Chem A4:3,615,1970)." Thus, I believe it is accurate to trace the first thinking about how a material should appropriately perform in an implant situation to about 1970. The nature of this "appropriate reaction" we call "biocompatibility" is expanded upon in the next section of this article.

Over the years, many definitions have been offered for biocompatibility. The most widely cited of these originated at a consensus conference on biomaterials held in Chester, UK in 1986. Biocompatibility is

"the ability of a material to perform with an appropriate host response in a specific application [2]"

Though accurate and philosophically useful, this definition provides no insights for how to assess biocompatibility or how to enhance it. An update of this definition was recently offered that acknowledges the biological reaction:

Biocompatibility "refers to the ability of a biomaterial to perform its desired function with respect to a medical therapy, without eliciting any undesirable local or systemic effects in the recipient or beneficiary of that therapy, but generating the most appropriate beneficial cellular or tissue response in that specific situation, and optimising the clinically relevant performance of that therapy [3]"

This definition, though a step in the right direction, does not address the most compelling concern with biocompatibility 
today - the reaction we call "biocompatible" seems quite the opposite of biocompatible. This point is elaborated upon in the last section of this article.

\section{Biocompatibility Today}

This word, biocompatibility, can be appreciated from a number of perspectives. We have considered, above, definitions from the literature. We can also address biocompatibility from the standpoint of the perceptions of physicians, the pronouncements of regulatory agencies, and the guidance of standards organizations.

How does the physician view biocompatibility? The observations that the physician makes when examining an explanted "biocompatible implant" are: (1) it is encased in a thin, tough collagen capsule, (2) the capsule is nonadherent to the implant, (3) there is little vascularity and little cellularity in the capsule, and (4) upon microscopic examination, macrophages and giant cells are seen at the surface of the implant, even years after implantation. These observations together are referred to as the foreign body reaction (FBR) [4]. ${ }^{1}$ There are many similarities to this observed reaction and to wound healing [5], the difference being that wounds resolve themselves and heal with no further reaction, while the evidence for ongoing, low-level inflammation persists indefinitely with implanted biomaterials.

The measurements that validate what we usually refer to as "biocompatibility" fall into two categories: (1) assessment of the substances that leach from the biomaterial or device and their effect on living cells or organisms and (2) measurement of the biological response to the biomaterials or device upon implantation in a living organism. The leachates from biomaterials are readily measured. A buffer soak of the biomaterial will extract such leachable substances (including processing additives, ions, manufacturing residues, retained solvents, low-molecular-weight material, and degradation products). If this buffer solution+leachate is added to live cells in culture, cell death will occur in those cases where cytotoxic extractables have been removed from the biomaterial. The direct relationship between cytotoxic leachates and cell viability was demonstrated long ago [6]. After more than 40 years of development, we now have reliable assays for leachates (for example, many of International Organization for Standardization (ISO) 10993 protocols) and we under-

\footnotetext{
${ }^{1}$ These reactions are seen for most soft tissue implants. They are not noted in avascular tissues such as cartilage and cornea or in the globe of the eye. In bone, the FBR reaction may be a precursor to the healing reaction we call osseointegration (see Ratner, B.D., $A$ Perspective on Titanium Biocompatibility, in Titanium in Medicine, D. Brunette et al., Editors. 2001, Springer Publishing, Inc.: Berlin. pp. 1-12.)
}

stand how to fabricate biomaterials with low levels (or zero) of extractables.

Subcutaneous, intramuscular, and other implantation tests look at the local, in vivo reaction to implants. Such tests are described in ISO 10993-6:2007 and in many publications. Samples for implantation must be sterile, endotoxin-free, and free of leachable substances. Such implants will, after approximately 1 month of implantation (typically in a mouse, rat, or rabbit), be encapsulated in a thin (approximately $100 \mu \mathrm{m}$ ), avascular, acellular, nonadherent collagenous bag. If the implantation site is relatively quiescent, and the reaction fits this description, the implant can be called "biocompatible." Such implants that have passed these in vitro and in vivo tests have been used with varying degrees of success in millions of humans.

This seems a good state of affairs. We can design materials to pass these tests. We can test in vitro and in vivo for "biocompatibility." But, here are the concerns. First, all materials free of endotoxin and leachables (polymers, metals, ceramics) give this similar reaction-we, the bioengineers, cannot modulate it or control it by, for example, changing the nature of the material. Second, the biological reaction is not one of "compatibility." It is a reaction of incompatibility, i.e., the body isolates the implant from itself with this tough, impermeable capsule. The consequences of this encapsulation are seen with implanted sensors that cease to sense body metabolites (for example, glucose) due the impermeable capsule, controlled drug delivery devices that no longer deliver drugs at the prescribed rate due to the permeation barrier surrounding them, recording electrodes that no longer have the intimate contact with nerves needed to record, breast implants that suffer from capsular contracture, pacemaker leads that are surrounded by scar and cannot be explanted, vascular grafts encased in a tough sheath that may inhibit compliance and healing, device-centered infection that might be related to low vascularity near the implant, etc. Most implant devices should function in an improved manner if they were healed into healthy, vascularized tissue, rather than entombed in "dead" scar.

\section{The Future of Biocompatibility}

Biocompatibility today, for materials with very low endotoxin levels and no measureable toxic leachables, is associated with a thin, stable foreign body capsule and only low levels of cellular reaction at the implant site. This description of biocompatibility is accepted by physicians, regulatory agencies, and standards organizations. In recent years, much effort has focused on developing technologies to reduce this capsule formation and to increase local vascularity surrounding implants. These methods aim to 
directly control the biology leading to the foreign body reaction, in contrast to simply accepting this reaction as the consequence or inevitable outcome of a biocompatible implant. This is driving the need for a new definition of biocompatibility,

One of the earliest efforts to thwart the FBR was associated with pacemaker leads. The foreign body reaction to electrical leads in the heart muscle results in a dense capsule that necessitated increasing the electrical energy of the pacing pulse (to penetrate the capsule) thus reducing potential battery life. A steroid-releasing electrode was developed to quiet the inflammatory reaction [7]. Dexamethasone binds to readily accessible, intracellular glucocorticoid receptors. It passively diffuses through cell walls and then forms a complex with the receptor protein that enters the cell nucleus and binds to DNA. The precise mechanism of action at this point is less clear, but the dexamethasone binding does downregulate factors associated with inflammation. Though this innovation that directly targets biological inflammation pathways has general success in reducing electrode fibrosis, the steroid inhibition of the FBR also inhibits normal healing and reconstruction. For this pacing application, mechanical anchoring in heart muscle seems satisfactory, and thus, normal healing is not required. Steroid delivery will not be a general solution to the problem of the FBR because of the overall inhibition of reconstructive healing.

Other approaches have been taken to address the FBR, scarring, and encapsulation. These include inhibition of specific proteins implicated in the FBR, modulation of inflammatory signals, the use of extracellular matrix (ECM) components (and decellularized ECM) to steer the inflammatory reaction toward a reconstructive outcome, tissue engineering approaches, and the use of porous structures to direct macrophages to a pro-healing pathway. For example, Zafirlukast, a leukotriene receptor antagonist, has been used to reduce capsular thickness in a model for breast implant encapsulation [8]. ADCON-T/N, a proprietary carbohydrate gel, has been shown to reduce neural scarring [9]. Plateletderived growth factor is an FDA-approved therapeutic for wound healing [10]. A study using knockout mice identified thrombospondin2 (TSP2) as a key regulator of the FBR [11]. By delivering antisense cDNA to block the production of TSP2, enhanced angiogenesis and a diffuse collagen deposition (in contrast to dense, highly aligned collagen in the expected capsule) were noted [12]. Very recent studies have suggested that inhibition of beta-arrestin can stop fibroblast invasion and reduce fibrosis and that microtubule stabilization can reduce scarring in damaged nerves $[13,14]$.

Tissue engineering approaches have also led to greatly improved biointegration and regeneration outcomes. Scaffolds seeded with cells, particularly when they are preconditioned in a bioreactor prior to implantation, often integrate into tissue in a vascularized, non-fibrotic manner. The scaffold without cells can heal in a fibrotic, avascular manner [15]. Though this is speculation, it may be the presence of cell-secreted ECM components that are transitioning the biomaterial scaffold from a "biocompatible" material generating the classic FBR to a structure that heals in a vascularized, reconstructive manner.

Perhaps, for reasons related to the ECM functionality considered above, decellularized ECM often heals in a regenerative manner upon implantation. Decellularized small intestinal submucosa (SIS), first used as a vascular prosthesis in 1989 [16], has been used successfully in numerous tissue sites (including vascular tissues, bladder, skin, dura mater, ligament, tendon, urethra, ureter, rotator cuff, and diaphragm) in more than one million human surgeries [17]. Similar results have been seen with other decellularized ECMs, for example, bladder. This description from the group that has pioneered SIS succinctly describes the unique biological reaction to this scaffold:

"Following implantation, host cells proliferate and differentiate into site-specific connective tissue structures, which appear to replace the SIS material within 90 days. The remodeling process has been strongly associated with angiogenesis, cell migration, and differentiation, and deposition of extensive extracellular matrix (ECM). The response is unique because the remodeled tissue is structurally and functionally similar to the injured tissue it was meant to replace [18]."

Two characteristics of the SIS biological reaction are important to mention here. First, a few days after implantation, the SIS is heavily infused with macrophages. Second, the degradation of the SIS structure precedes the formation of new tissue. The degradation products of SIS (peptide fragments) are highly chemoattractive to cells in vitro and pro-regenerative in vivo [19].

In recent years, our research group has been exploring a class of porous scaffolds that have interesting parallels to the decellularized ECMs in their in vivo bioreaction. The work was stimulated by almost 40 years of observation that the FBR to an implanted material is modulated by roughness, porosity, and/or texture [20-23]. Our porous scaffold materials, prepared by sphere templating, are characterized by a single pore size (in contrast to a pore size distribution), with pores spherical in shape and interconnected. This pore structure allowed us to accurately assess the effect of pore size on the FBR. We found that when the pores were $30-40 \mu \mathrm{m}$ in diameter, there was considerable angiogenesis and much reduced capsule formation (loose, open collagen fibrils, in contrast to highly oriented, dense collagen) [24-27]. When pores were larger or smaller, the classic FBR was observed. This effect is independent of biomaterial (to date, we have seen this with 
hydrogel, silicone elastomer, and polyurethane). Also, surface modification of the hydrogel with collagen I or with laminin I had little effect on the healing phenomenon. Regenerative healing has been noted in skin (subcutaneously and percutaneously) in sclera, in heart, in skeletal muscle, and in bone.

We consistently observe that the sphere-templated polymers are heavily infused with macrophages during the healing process, similar to the decellularized ECMs. However, these sphere-templated polymers do not degrade to bioactive fragments like SIS. We believe they function by mechanically guiding the macrophage, because of the confinement within the pores, to a pro-regenerative phenotype (often called M2) in contrast to a pro-inflammatory phenotype (M1) $[25,28,29]$. In the case of the SIS and sphere-templated polymer, by controlling the fate of the macrophage, the FBR is deflected, and regenerative healing occurs.

\section{The Manifesto and a Biocompatibility Definition for the Twenty-first Century}

\section{Today's definition of biocompatibility, \\ "the ability of a material to perform with an appropriate host response in a specific application [2]"}

refers to a reaction where the body isolates the "biocompatible" implant from itself in a tough, fibrous bag. This reaction appears to me to be a "bio-incompatibility" between implant and organism. In contrast, using some of the strategies described in the previous section of this article, implants can be made to heal into the body in a vascularized, non-fibrotic fashion and with considerable reconstruction of the tissue into which the implant was placed.

As an example of these contrasting biological reactivities, a solid slab of poly(2-hydroxyethyl methacrylate) hydrogel can be implanted in an animal side by side with a spheretemplated $30-\mu \mathrm{m}$ porous structure of the same polymer. The solid slab will heal with the classic FBR, a dense capsule surrounding the implant. The same polymer with $30-\mu \mathrm{m}$ pores will show little or no fibrosis, excellent vascularization, and reconstruction of the original tissue [25]. We have in the two specimens described here the same biomaterial used in the same specific application. The biological reactions are diametrically different. How can we label both reactions "biocompatible?"

The ability to control the host biological reaction with precision has evolved remarkably since the 1970s when the word biocompatibility was first used. This control is based on a vastly enhanced understanding of the biological mechanisms associated with inflammation and healingwe can use that knowledge in an engineering sense to facilitate healing and integration.
For these reasons, I put forth this public declaration, this manifesto, expressing concerns with the ways we think about and use biocompatibility. I offer this definition of biocompatibility to be more consistent with our knowledge of biological reactions and our recently discovered ability to manipulate the reaction:

"BIOCOMPATIBILITY: the ability of a material to locally trigger and guide non-fibrotic wound healing, reconstruction and tissue integration"

In contrast, I offer this definition for the biomaterials that are widely used today in medicine, largely with satisfactory outcomes:

"BIOTOLERABILITY: the ability of a material to reside in the body for long periods of time with only low degrees of inflammatory reaction"

Definition has been defined as "the act of making definite, distinct or clear." These two definitions clarify the performance of implanted biomaterials: (1) the new possibilities for integrative, reconstructive performance and (2) the traditional performance that has served us adequately for 40 or more years.

This manifesto calls for the adoption of definitions for biocompatibility that are meaningful in the twenty-first century and will help to clarify biomaterial performance to physicians, patients, researchers, and regulatory agencies. Such clarity can be realized using the principles and discoveries from modern mechanistic, quantitative biology.

Acknowledgments I gratefully acknowledge the support I have received from the National Science Foundation (University of Washington Engineered Biomaterials Engineering Research Center) and from the National Heart, Lung and Blood Institute (R01 HL064387) that has helped formulate many of the ideas expressed here. Many colleagues over the years have also contributed to my thinking on this subject including Russell Ross, Lester Sauvage, Allan Hoffman, Tom Horbett, David Williams, Andreas von Recum, James Anderson, Monty Reichert, and Steve Badylak.

\section{References}

1. Bing, J. (1955). The tissue reaction to implanted plastics. Acta Pathologica et Microbiologica Scandinavica Supplementum, 105, $16-26$.

2. Williams, D. F. (1987). Definitions in biomaterials. Progress in biomedical engineering, 4 (p. 72). Amsterdam: Elsevier.

3. Williams, D. F. (2008). On the mechanisms of biocompatibility. Biomaterials, 29(20), 2941-2953.

4. Anderson, J. M., Rodriguez, A., \& Chang, D. T. (2008). Foreign body reaction to biomaterials. Seminars in Immunology, 20, 86100.

5. Ross, R. (1970). Wound healing — a review. In E. A. Balazs (Ed.), Chemistry and molecular biology of the intercellular matrix. Structural organization and function of the matrix (Vol. 3, pp. 1739-1751). New York: Academic Press. 
6. Homsy, C. A. (1970). Bio-compatibility in selection of materials for implantation. Journal of Biomedical Materials Research, 4, 341-356.

7. Mond, H., Stokes, K., Helland, J., Grigg, L., Kertes, P., Pate, B., et al. (1988). The porous titanium steroid eluting electrode: a double blind study assessing the stimulation threshold effects of steroid. Pacing and Clinical Electrophysiology, 11(2), 214-219.

8. Spano, A., Palmieri, B., Palmizi Taidelli, T., \& Nava, M. B. (2008). Reduction of capsular thickness around silicone breast implants by zafirlukast in rats. European Surgical Research, 41(1), $8-14$.

9. Petersen, J., Russell, L., Andrus, K., MacKinnon, M., Silver, J., \& Kliot, M. (1996). Reduction of extraneural scarring by ADCON-T/N after surgical intervention. Neurosurgery, 38(5), 976-983.

10. Barrientos, S., Stojadinovic, O., Golinko, M. S., Brem, H., \& Tomic-Canic, M. (2008). PERSPECTIVE ARTICLE: Growth factors and cytokines in wound healing. Wound Repair and Regeneration, 16(5), 585-601.

11. Kyriakides, T. R., Leach, K. J., Hoffman, A. S., Ratner, B. D., \& Bornstein, P. (1999). Mice that lack the angiogenesis inhibitor, thrombospondin 2, mount an altered foreign body reaction characterized by increased vascularity. Proceedings of the National Academy of Science of the United States of America, 96, 1-6.

12. Kyriakides, T. R., Hartzel, T., Huynh, G., \& Bornstein, P. (2001). Regulation of angiogenesis and matrix remodeling by localized, matrix- mediated antisense gene delivery. Molecular Therapy, 3 (6), 842-849.

13. Hellal, F., Hurtado, A., Ruschel, J., Flynn, K. C., Laskowski, C. J., Umlauf, M., et al. (2011). Microtubule stabilization reduces scarring and causes axon regeneration after spinal cord injury. Science, 331(6019), 928-931.

14. Lovgren, A. K., Kovacs, J. J., Xie, T., Potts, E. N., Li, Y., Foster, W. M., et al. (2011). b-Arrestin deficiency protects against pulmonary fibrosis in mice and prevents fibroblast invasion of extracellular matrix. Science Translational Medicine, 3(74), 74ra23-74ra23.

15. De Filipo, R. E., Yoo, J. J., \& Atala, A. (2002). Urethral replacement using cell seeded tubularized collagen matrices. The Journal of Urology, 168, 1789-1793.

16. Badylak, S. F., Lantz, G. C., Coffey, A., \& Geddes, L. A. (1989). Small intestinal submucosa as a large diameter vascular graft in the dog. Journal of Surgical Research, 47(1), 74-80.

17. Badylak, S. F. (2007). The extracellular matrix as a biologic scaffold material. Biomaterials, 28, 3587-3593.

18. Hodde, J. P., Badylak, S. F., Brightman, A. O., \& Vytik-Harbin, S. L. (1996). Glycosaminoglycan content of small intestinal submucosa: a bioscaffold for tissue replacement. Tissue Engineering, 2(3), 209217.

19. Vorotnikova, E., McIntosh, D., Dewilde, A., Zhang, J., Reing, J. E., Zhang, L., et al. (2010). Extracellular matrix-derived products modulate endothelial and progenitor cell migration and proliferation in vitro and stimulate regenerative healing in vivo. Matrix Biology, 29(8), 690-700.

20. Karp, R. D., Johnson, K. H., Buoen, L. C., Ghobrial, H. K. G., Brand, I., \& Brand, K. G. (1973). Tumorigenesis by Millipore filters in mice: histology and ultrastructure of tissue reactions as related to pore size. Journal of the National Cancer Institute, 51 (4), 1275-1279.

21. Recum, A. F., Shannon, C. E., Cannon, C. E., Long, K. J., Kooten, T. G., \& Meyle, J. (1996). Surface roughness, porosity, and texture as modifiers of cellular adhesion. Tissue Engineering, 2(4), 241-253.

22. Brauker, J. H., Carr-Brendel, V. E., Martinson, L. A., Crudele, J., Johnston, W. D., \& Johnson, R. C. (1995). Neovascularization of synthetic membranes directed by membrane microarchitecture. Journal of Biomedical Materials Research, 29, 1517-1524.

23. Clowes, A. W., Kirkman, T. R., \& Reidy, M. A. (1986). Mechanisms of arterial graft healing - rapid transmural capillary ingrowth provides a source of intimal endothelium and smooth muscle in porous PTFE prostheses. American Journal of Pathology, 123(2), 220-230.

24. Marshall, A. J., Irvin, C. A., Barker, T., Sage, E. H., Hauch, K. D., \& Ratner, B. D. (2004). Biomaterials with tightly controlled pore size that promote vascular in-growth. ACS Polymer Preprints, 45 (2), 100-101.

25. Madden, L. R., Mortisen, D. J., Sussman, E. M., Dupras, S. K., Fugate, J. A., Cuy, J. L., et al. (2010). Proangiogenic scaffolds as functional templates for cardiac tissue engineering. Proceedings of the National Academy of Sciences of the United States of America, 107(34), 15211-15216.

26. Fukano, Y., Usui, M. L., Underwood, R. A., Isenhath, S., Marshall, A. J., Hauch, K. D., et al. (2010). Epidermal and dermal integration into sphere-templated porous poly(2-hydroxyethyl methacrylate) implants in mice. Journal of Biomedical Materials Research. Part A, 94(4), 1172-1186.

27. Ratner, B., \& Atzet, S. (2009). Hydrogels for healing. In R. Barbucci (Ed.), Hydrogels (pp. 43-51). Milan: Springer.

28. Mantovani, A. (2006). Macrophage diversity and polarization: in vivo veritas. Blood, 108(2), 408-409.

29. Badylak, S., Valentin, J., Ravindra, A., Mccabe, G., \& StewartAkers, A. (2008). Macrophage phenotype as a determinant of biologic scaffold remodeling. Tissue Engineering. Part A, 14(11), $1835-1842$. 